\title{
Perceptual equivalence between visual and tactual pattern perception: An anchoring study'
}

\author{
STANLEY H. BRUMAGHIM, THE BOEING COMPANY \\ D. R. BROWN, ${ }^{2}$ PURDUE UNIVERSITY
}

An anchoring design was used to investigate correspondence between visual and tactual scaling of complexity when two samples of random polygons served as stimulus forms. The results support the conclusion that within an adjustment for differential acuity between vision and active touch input is referred to a common perceptual mechanism.

A number of experimental approaches have been directed toward demonstrating that input from the various sensory modalities is referred to a common perceptual mechanism. These have included demonstrations of intermodal transfer of learning (e.g., Bjorkman, Garvill, \& Molander, 1965), cross-modal matching (Stevens, Mack, \& Stevens, 1960), intermodal recognition (Caviness \& Gibson, 1964; Lobb, 1965), and intermodal anchoring (Behar \& Bevan, 1961; Goldstone, Boardman, \& Lhamon, 1959; Goldstone \& Goldfarb, 1963).

In earlier studies (Brown \& Brumaghim, 1968; Owen \& Brown, 1968), it was emphasized that the study of perceptual equivalence raises the question of stimulus equivalence. If equivalent responses occur to stimulation presented to different modalities, the question of what stimulus attributes account for the equivalence arises. This latter question remains almost totally unanswered in view of the difficulties associated with specifying a perceptuallyrelevant physics for complex stimulus situations. In view of progress in developing the psychophysical study of form perception (Attneave \& Arnoult, 1956; Behrman \& Brown, 1968; Brown \& Andrews, 1968; Brown \& Owen, 1967), it is possible, however, to systematically study such questions for modalities in which form perception is meaningful. In pattern perception positive transfer of learning has been demonstrated between visual and tactual conditions of presentation (e.g., Gaydos, 1956; Semmes, Weinstein, Ghent, \& Teuber, 1954), intermodal matching has been demonstrated (Caviness \& Gibson, 1964; Lobb, 1965) and some success in relating visual and tactual complexity judgments to a common set of pattern attributes has been reported (Brown \& Brumaghim, 1968; Owen \& Brown, 1968). This study extends previous work by demonstrating that cross-modal anchoring effects occur in visual and tactual pattern perception.

\section{METHOD}

Subjects

Eighty university students, $\mathbf{4 0}$ males and $\mathbf{4 0}$ females, served as Ss. Of these, 17 served to satisfy partial requirements of an introductory psychology course and each of 63 was paid $\$ 2.00$ for participation.

\section{Patterns}

Two samples of 10 random polygons, designated Set $A$ and Set B, were selected from previous research (Owen \& Brown, 1968) to be approximately equidistant on a unidimensional visual complexity continuum.

Patterns were generated in a 100 by 100 unit matrix according to a modified Method I of Attneave and Arnoult (1956) with a single modification of these rules to assure a specified $(4,8,12$, 16 , or 20) number of independent sides. All forms were equated for area at $1250 \mathrm{sq}$ units and were geometrically centered on 100 $x 100 \mathrm{~mm}$ white posterboard squares on which they were presented. Forms were cut from black, finely-graded sandpaper (Behr-Manning 360A) and glued to the posterboard squares. These forms, shown in Fig. 1 with their complexity scale values, were used in a previous study (Brown \& Brumaghim, 1968) and a detailed discussion of the stimulus domain from which they were sampled has been presented elsewhere (Brown \& Owen, 1967).

\section{Apparatus and Procedure}

The apparatus, which has been described elsewhere (Owen \& Brown, 1968), consisted essentially of a Plexiglas display surface for presenting forms tactually and visually, circuitry for measuring latency, and peripheral equipment for shielding forms from sight during tactual presentation and between trials.

The experiment was carried out in two sessions for all Ss: in the first session, forms were unidimensionally scaled for complexity and in the second session forms were rescaled in the presence of anchors. In the first session, the 80 Ss were divided into groups (10 males and 10 females) by randomly assigning them to four conditions which were defined by the factorial combination of two levels of form complexity (Low and High) and two modes of presentation (Visual and Tactual). Ten low-complexity forms were obtained by selecting the five forms from Set $A\left(A_{1}, A_{2}, A_{3}, A_{4}\right.$, $\left.A_{6}\right)$ and Set $B\left(B_{1}, B_{2}, B_{3}, B_{4}, B_{6}\right)$ with the lowest complexity scale values obtained in a previous study (Owen \& Brown, 1968). Similarly, the 10 high-complexity forms were selected $\left(A_{6}-A_{10}\right.$ and $B_{6}-B_{10}$ ) from the remainder of the scale with form $A_{6}$ and $B_{6}$ shared by both high and low-complexity forms. For both visual and tactual presentation, each of the 10 forms was presented three times so that each $S$ received 30 trials with the order of forms randomized within blocks of 10 trials.

For both visual and tactual ratings, Ss were asked to assigit numbers from " 1 " (lowest complexity) to " 9 " (highest complexity) to each form and 10 practice trials with the appropriate forms preceded experimental testing. For groups receiving visual presentations, $S$ was seated across a table from $E$ and was told that his rating latency would be measured but that $E$ was interested in his normal reaction time. On each trial, a curtain was drawn in front of the apparatus. When E presented a ready light, the curtain was withdrawn, $S$ released a depressed panel to start timing the trial, and the rating spoken into a voice key terminated the timed trial. For tactual presentations, the same procedure was followed except that Ss explored forms behind the curtain which remained drawn.

\begin{tabular}{|c|c|c|c|}
\hline SHAPE & $\begin{array}{l}\text { COMPLEXITY } \\
\text { SCALE VALUES } \\
\end{array}$ & SHAPE & $\begin{array}{l}\text { COMPLEXITY } \\
\text { SCALE VALUES } \\
\end{array}$ \\
\hline$A_{1}$ & 1.75 & $B_{1}$ & 1.75 \\
\hline$A_{2}$ & 2.60 & $B_{2}$ & 2.67 \\
\hline$A_{3}$ & 3.22 & $B_{3}$ & 3.18 \\
\hline$A_{4}$ & 3.75 & $B_{4}$ & 3.70 \\
\hline$A_{5}$ & 4.25 & $B_{5}$ & 4.28 \\
\hline$A_{6}$ & 4.65 & $B_{6}$ & 4.72 \\
\hline$A_{7}$ & 5.12 & $B_{7}$ & 4.98 \\
\hline$A_{8}$ & 5.50 & $\mathrm{~B}_{\boldsymbol{8}}$ & 5.55 \\
\hline$A_{9}$ & 6.18 & $\mathrm{~B}_{9}$ & 6.15 \\
\hline$A_{10}$ & 6.68 & $B_{10}$ & 6.68 \\
\hline
\end{tabular}

Fig. 1. The two sets of shapes and their visual complexity scale values. 
They were free to explore the forms in any manner which did not require moving them from their position in the apparatus.

In the second session, Ss again rated the same forms they had rated in the first session (standard forms), but in the presence of anchoring forms. Eight groups of $10 \mathrm{Ss}$ each (five male and five female) were formed by dividing the four groups from Session 1 on the basis of the modality in which anchoring forms were introduced. Both intramodal and intermodal anchors were used and the standard forms were presented in the same modality as they had been in Session 1 for each $S$. These eight groups were defined, then, by the factorial combination of High and Low complexity forms, visual and tactual presentation of standard forms, and visual and tactual presentation of anchoring forms. The low-complexity forms within each set were anchored by the five high-complexity forms in the same set (A or B); the five high-complexity forms within each set were anchored by the five low-complexity forms in the same set. With this restriction, 25 trials were defined by combining all possible standards and anchors within Set $A$ and 25 trials were defined by these combinations for Set B. Ss in all groups received the 50 trials with the order of pair presentations determined randomly.

The procedure for Session 2 was similar to that used in the first session except that judgments were made in the presence of anchors. On trials in which high-complexity forms anchored low-complexity standards, Ss were instructed to assign " 9 " to the anchoring form; low-complexity anchors for high-complexity standards were assigned " 1 " as a scale value. Ss were given five practice trials, were told to make their ratings as independently as possible across trials, and no attempt was made to influence the distribution of ratings. Standard forms were presented to the left of anchor forms under all conditions. Under tactual-tactual conditions, Ss explored the left anchor form and then the right standard form with their preferred hand and were free to examine both forms repeatedly. Under tactual anchor-visual standard, visual anchor-tactual standard, and visual anchor-visual standard

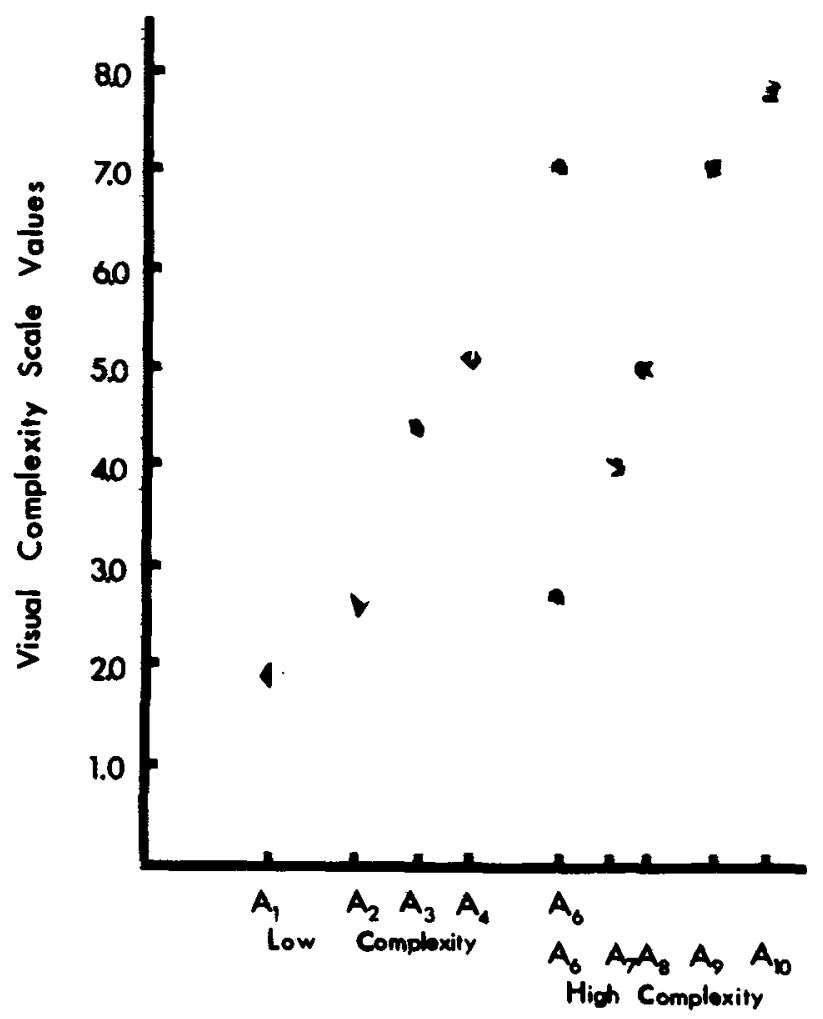

Fig. 2a. Relation between the visual complexity scale and mean visual ratings for Set $A$ forms scaled visually.

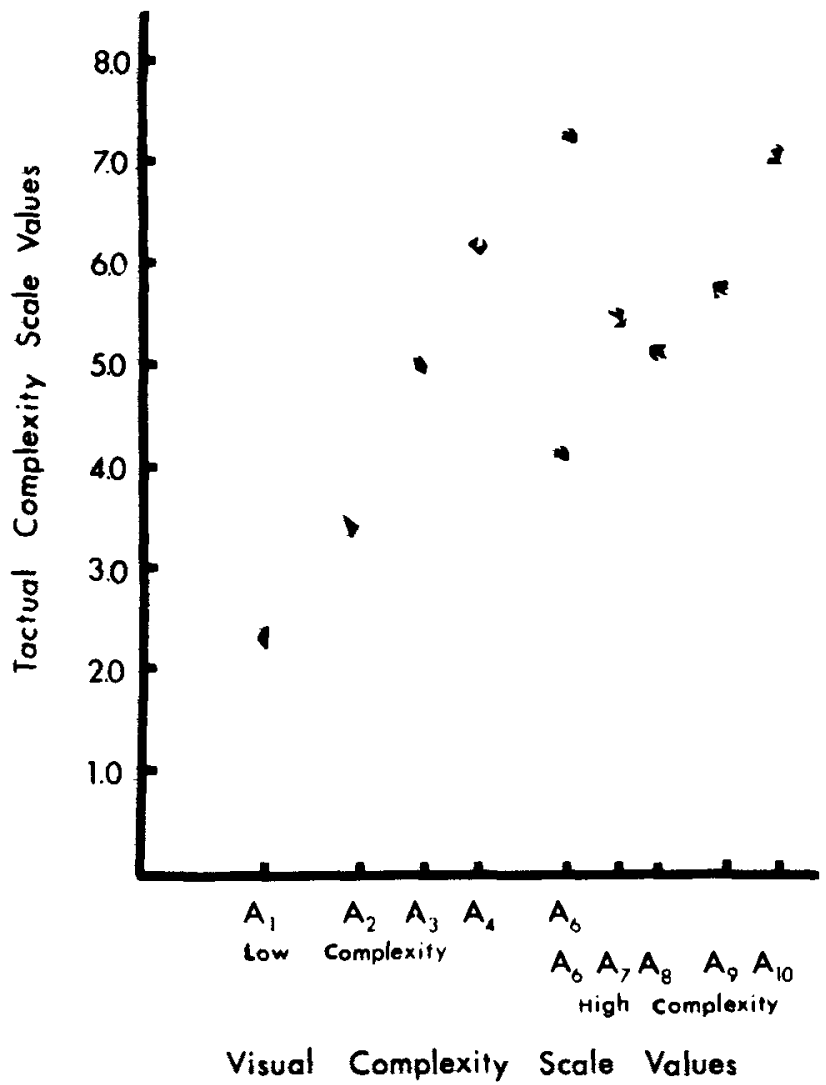

Fig. 2b. Relation between the visual complexity scale and mean visual ratings for Set $A$ forms scaled tactually.

conditions, exploration of both the standard and anchor forms occurred simultaneously.

\section{RESULTS}

The scaling data from Session 1 were examined in terms of their reliability and in terms of their agreement with previously-obtained results. Intra-class correlations were used to estimate reliabilities within the four experimental groups and were found to be $.85, .86, .85$, and .54 for low-complexity visual and tactual ratings and high-complexity visual and tactual ratings respectively. The relatively low reliability of tactual ratings of complex forms will be discussed below.

Mean ratings over Ss and trials were used for scale values and are summarized for the four conditions in Fig. 2a-2d. In those figures, the abscissa values are those which were used from an earlier study (Owen \& Brown, 1968) to select the shapes. The ordinate values are those obtained herein. In reading these graphs, it is well to keep in mind that different groups of Ss scaled the low- and high-complexity shapes. Clearly, both the tactual and visual data are in close linear agreement with the previously-obtained results. It is also clear that tactual and visual scale values are very highly related. Finally, since the shapes are ordered in pairs on the basis of the number of independent sides, both tactual and visual complexity ratings are linearly predictable from the number of independent sides.

Analyses of the transformed latency scores also supported previous results. There was a large modality main effect. The visual mean latencies were $1.52 \mathrm{sec}$ and $1.85 \mathrm{sec}$ for low- and high-complexity forms respectively. The corresponding tactual mean latencies were $14.50 \mathrm{sec}$ and $14.47 \mathrm{sec}$. Moreover, the tactual latencies were linear with a positive slope over the complexity scale while the visual latencies were quadratic over the scale with a peak in the middle of the scale and shorter latencies at both scale extremes. 


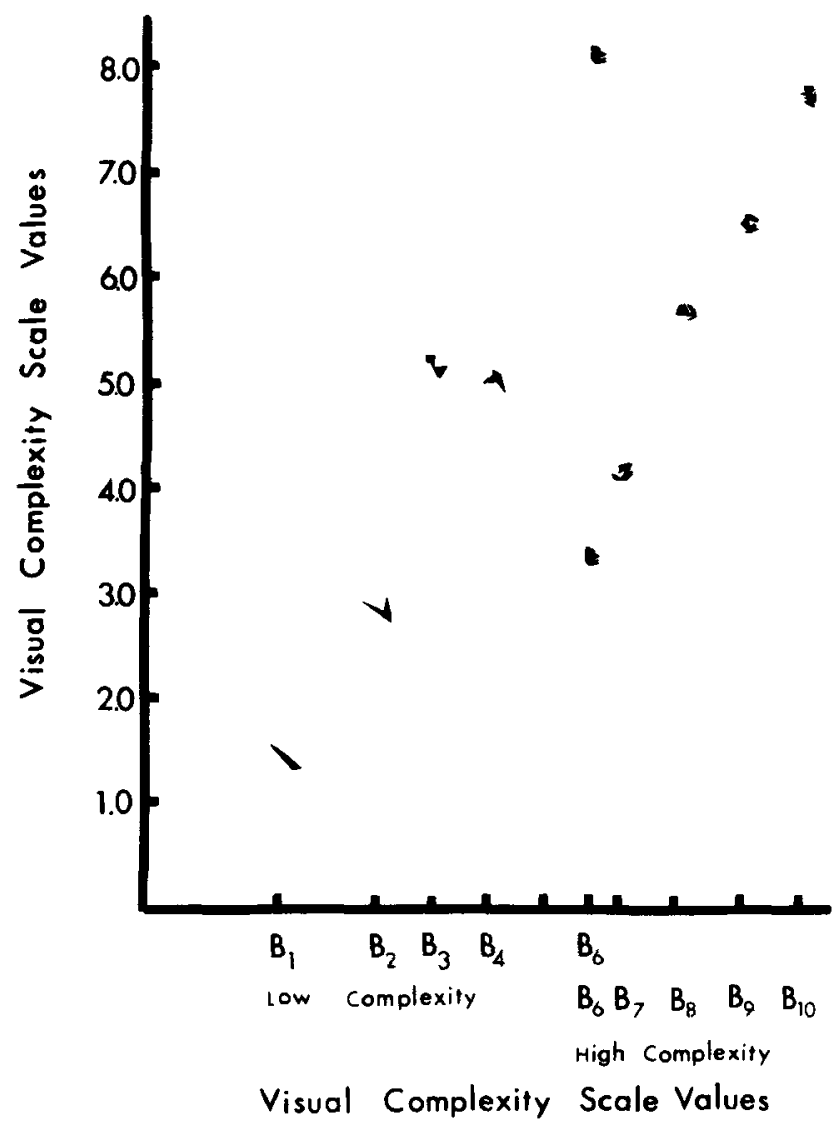

Fig. 2c. Relation between the visual complexity scale and mean visual ratings for Set B forms scaled visually.

Since the results from the first session were reliable and highly similar to previous results with these forms, the data from the second session were examined for inter- and intramodal anchoring effects. These data are summarized in Fig. 3a-3d where each figure summarizes the data for two of the eight experimental conditions. Anchoring effects are typically described as "assimilation" effects when ratings are shifted toward the value of anchoring stimuli and "contrast" effects when ratings are shifted away from anchors. Fig. 3a-3d make it apparent that both types of effects occurred.

In those figures, the shift for an entire set of five standard forms is shown for each anchor form used in that condition. The ordinate values were computed as a per cent of the possible shift from the median of the Session 1 ratings. Thus, if the median from Session 1 were 4.5 , the anchored median could shift from 4.5 to 1 or from 4.5 to 9 . The degree of anchoring and its direction were computed as a per cent of this total possible shift under each condition.

When attention is restricted to the effects of high-complexity anchors upon the ratings of low-complexity forms (Fig. 3a-3b) an interesting type of asymmetry of effects is apparent. In Fig: 3a, the effects of visual anchors on both visual and tactual ratings and of tactual anchors on tactual ratings are very similar. When the anchor was similar to the series being rated (low complexity) assimilation occurred, but as the standard and anchor series became less similar contrast effects occurred. However, when tactual anchors were combined with visual standards assimilation effects occurred. Similarly, for Set B (Fig. 3b) the functions (no effects changing to contrast effects) were similar under all conditions except that tactual anchors again created assimilation effects with visual ratings.

The results when low-complexity anchors were presented with standards of high complexity (Fig. 3c-3d) were highly consistent under all conditions. Minor contrast occurred with the most deviant anchor, assimilation effects were obtained otherwise, and the effects of tactual and visual anchors were very similar.

\section{DISCUSSION}

The Session 1 data are very clear and support the general conclusion that complexity judgments are very similar for visual and tactual conditions of exploration. The difference in shape of the functions relating latency to the complexity scale for the two modalities has been discussed (Owen \& Brown, 1968). It was suggested that tactual latencies largely reflect information gathering which increases linearly, as complexity increases. The visual latency function, in contrast, largely reflects information processing where the reduction in latency at both extremes may indicate that stimuli need be compared to fewer "ideals."

The anchoring data require differential interpretation for lowand for high-complexity anchors. When anchors were of low complexity, the anchoring effects were highly similar both within and across modalities. When anchors were of high complexity, however, the tactual anchoring of visual ratings created assimilation effects where consistent mixed effects occurred under all other conditions of anchoring. This asymmetry, coupled with the low reliability of tactual ratings of complex shapes, increased latency of tactual ratings with increased complexity, and the fact that tactual ratings at the high end of the scale were lower than visual ratings under comparable conditions, all suggest that this anchoring difference merely reflects an intermodal acuity difference. Stated simply, if the tactual forms were adjusted for the size of detail to be comparable to visual acuity, these differences would disappear and the results would be consistent with the rest of the data which provide very strong support for a single perceiver.

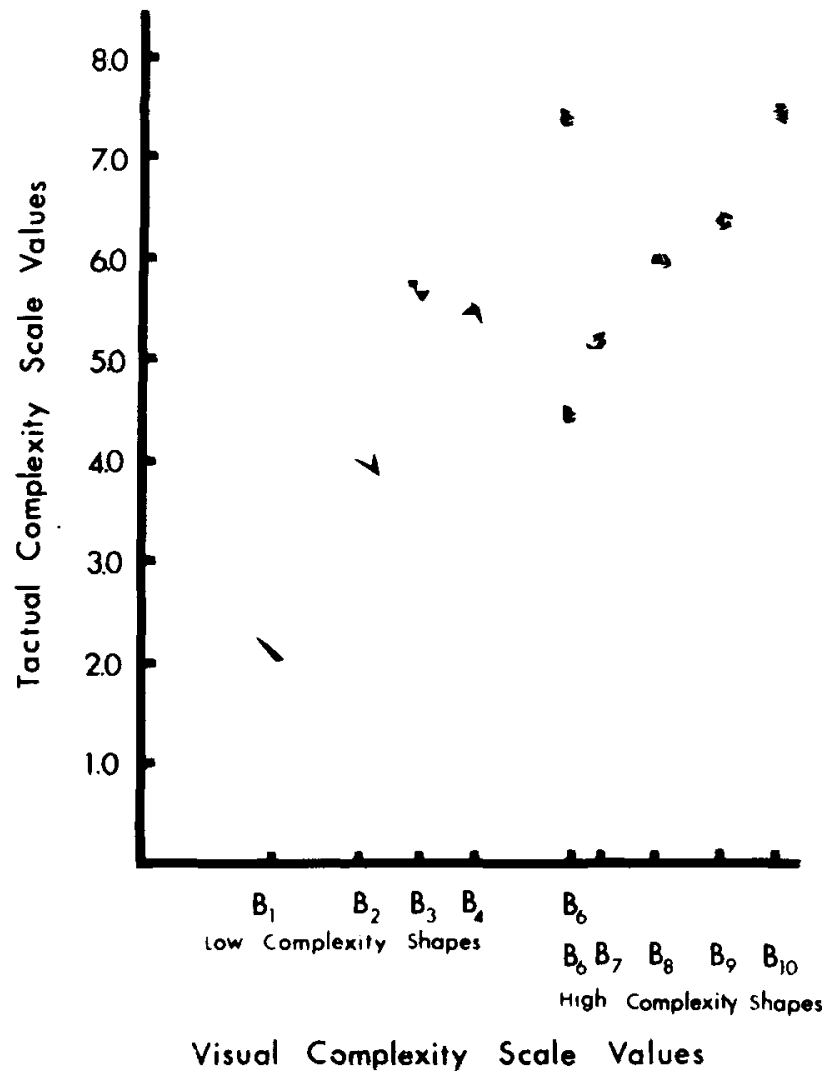

Fig. 2d. Relation between the visual complexity scale and mean visual ratings for Set B forms scaled tactually. 


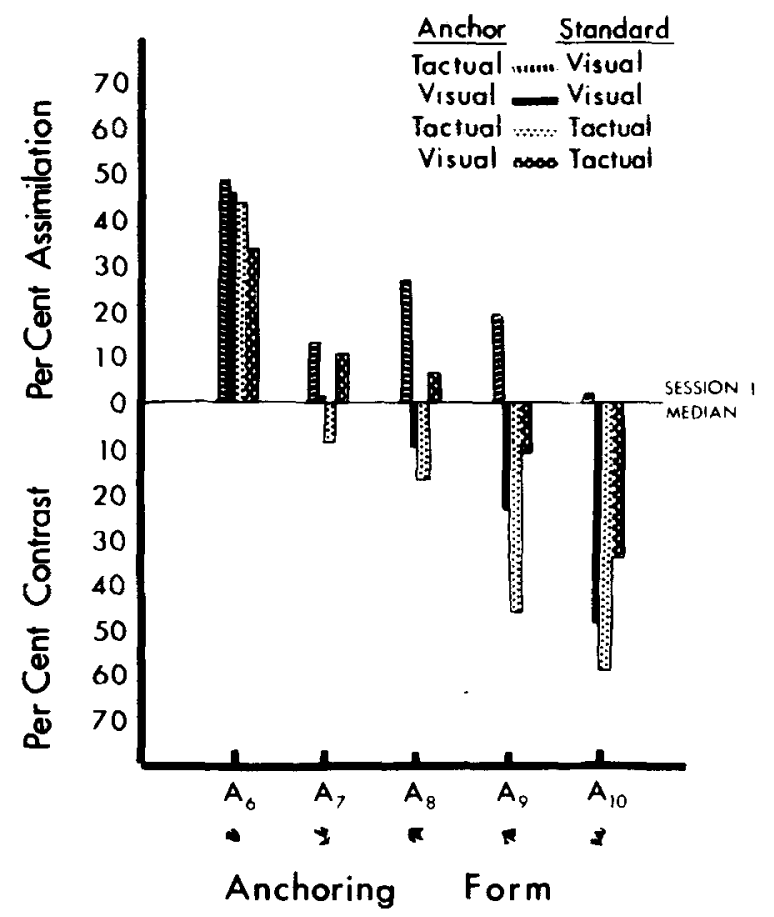

Fig. 3a. Anchor effects when Set A, high complexity shapes were used under four conditions of scaling.

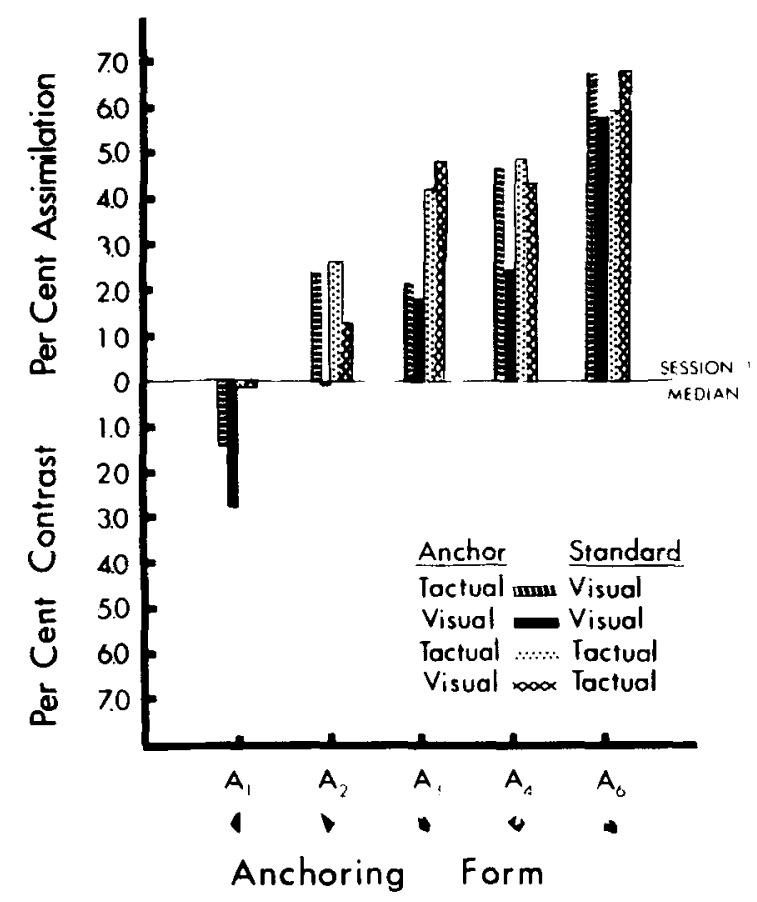

Fig. 3c. Anchor effects when Set A, low complexity dapes were used under four conditions of scaling.

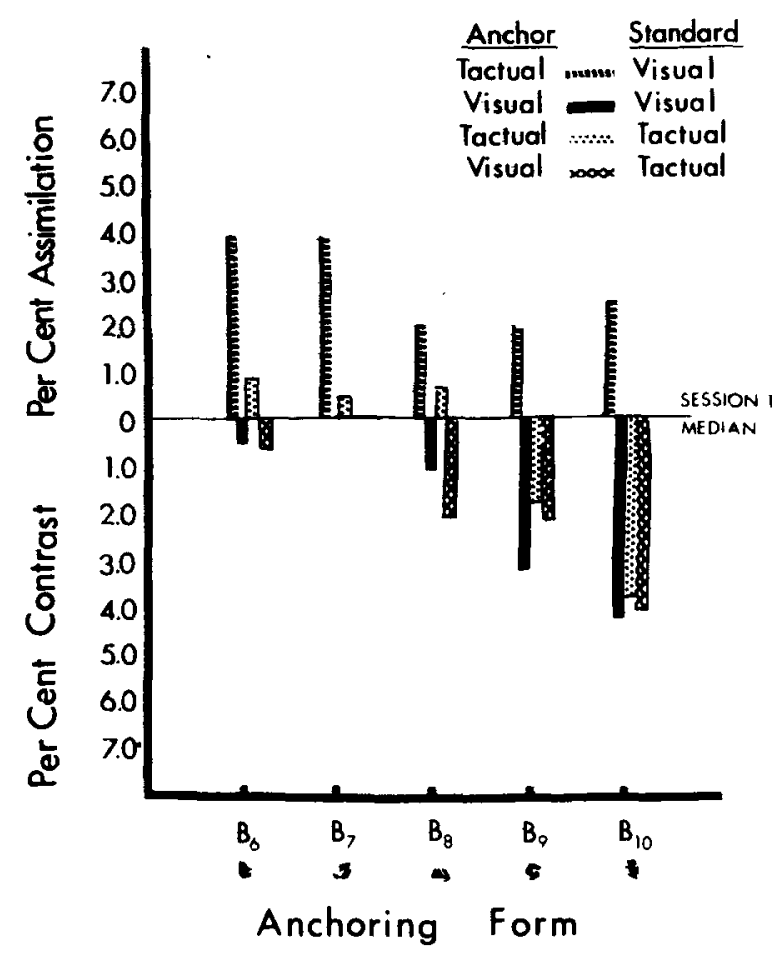

Fig. 3b. Anchor effects when Set B, high complexity shapes were used under four conditions of scaling.

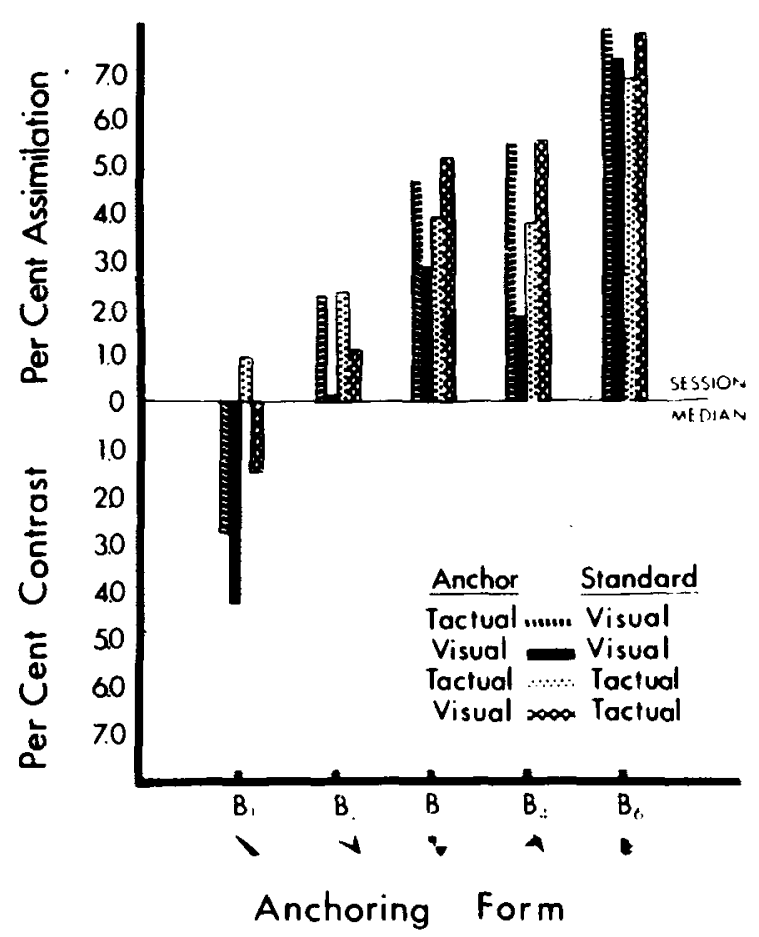

Fig. 3d. Anchor effects when Set B, low complexity shapes were used under four conditions of scaling. 


\section{REFERENCES}

ATTNEAVE, F., \& ARNOULT, M. D. The quantitative study of shape and pattern perception. Psychol. Bull, 1956, 53, 452-471.

BEHAR, E. J., \& BEVAN, W. The perceived duration of auditory and visual intervals: Cross-modal comparison and interaction. Amer. J. Psychol., $1961,74,17-26$.

BEHRMAN, B. W., \& BROWN, D. R. Multidimensional scaling of form. Percept. \& Psychophys., 1968, in press.

BJORKMAN, M., GARVILL, J., \& MOLANDER, B. Cross-modal transfer as a function of preparatory set and distinctiveness of stimulus aspects. Reports from the Psychological Laboratories, University of Stockholm, 1965, No. 186.

BROWN, D. R., \& ANDREWS, M. H. A multidimensional analysis of visual shape discrimination. Percept. \& Psychophys., 1968, in press.

BROWN, D. R., \& BRUMAGHIM, S. H. Perceptual equivalence, pattem perception, and multidimensional psychophysics. Submitted to Percept. \& Psychophys, 1968.

BROWN, D. R., \& OWEN, D. H. The metrics of visual form: Methodological dyspepsia. Psychol. Bull., 1967, 68, 243-259.

CAVINESS, J. A., \& GIBSON, J. J. The equivalence of visual and tactual stimulation for the perception of solid forms. Paper presented at Eastern Psychological Association, Atlantic City, April, 1962.

GAYDOS, H. F. Intersensory transfer in the discrimination of form. Amer. J. Psychol, 1956, 69, 107-110.
GOLDSTONE, S., BOARDMAN, W. K., \& LHAMON, W. T. Inter-sensory comparisons of temporal judgments. J. exp. Psychol, 1959, 57, 243-248.

GOLDSTONE, S., \& GOLDFARB, J. L. Judgment of filled and unfilled durations: Intersensory factors. Pencept. mot. Skills, 1963, 17, 763-774.

LOBB, H. Vision versus touch in form discrimination. Canad. J. Psychol, $1965,19,175-187$.

OWEN, D. H., \& BROWN, D. R. Visual and tactual form complexity: A psychophysical approach to perceptual equivalence. Submitted to J. exp. Psychol., 1968.

SEMMES, J., WEINSTEIN, S., GHENT, L., \& TEUBER, H. Performance on complex tactual tasks after brain injury in man. Amer. J. Psychol, 1954, 67, 220-240.

STEVENS, J. C., MACK, J. D., \& STEVENS, S. S. Growth of sensation on seven continua as measured by force of handgrip. J. exp. Psychol., 1960, $59,60-67$.

\section{NOTES}

1. This research was supported by Research Grant HD-00909 from the National Institute of Child Health and Human Development.

2. Address: Department of Psychology, Purdue University, Lafayette, Indiana 47907.

(Accepted for publication May 21, 1968.) 